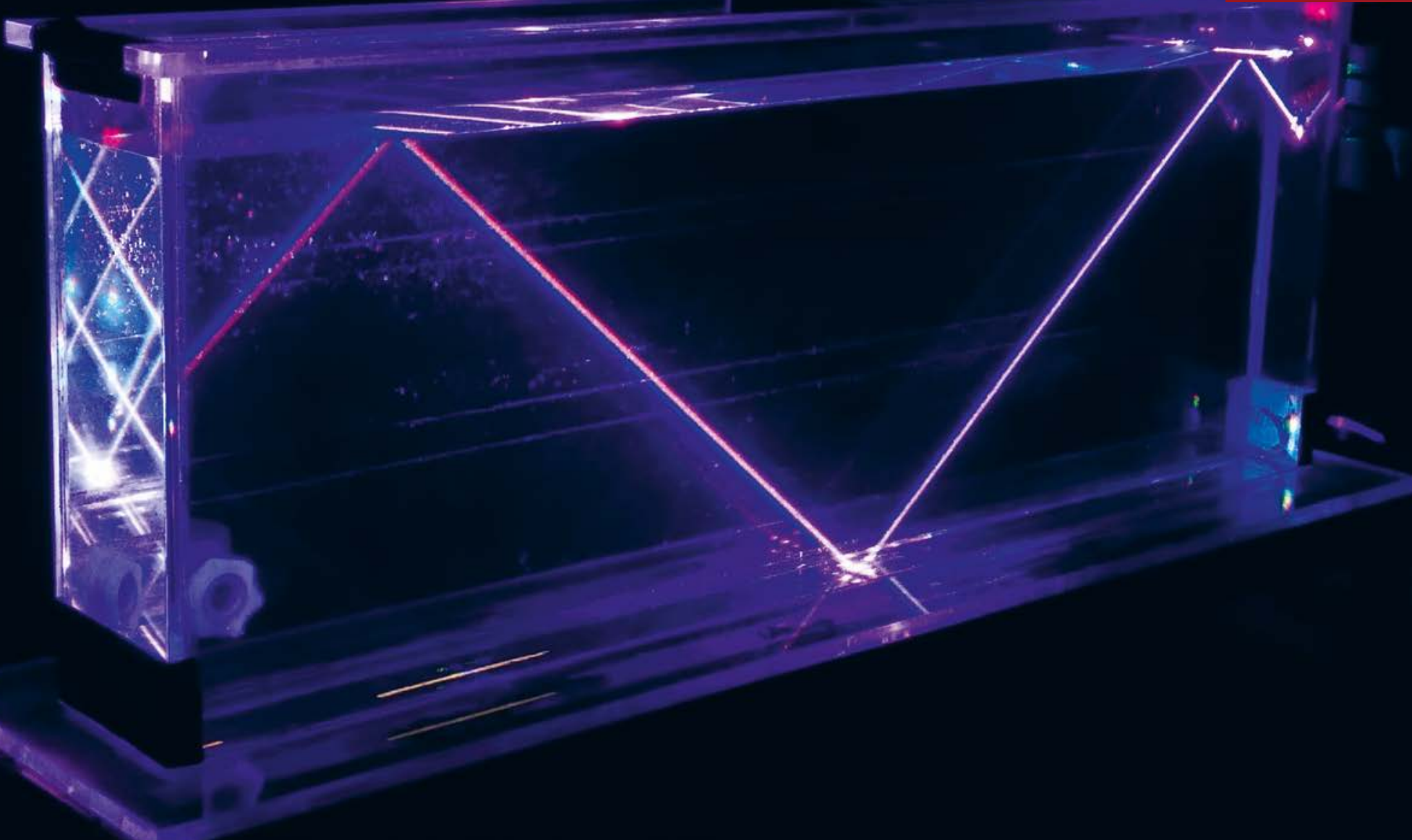

\title{
A NOBEL CAUSE: PUBLIC ENGAGEMENT AND OUTREACH
}

. Christopher Holmes, Pearl John and Callum Stirling - Dol: https://doi.org/10.1051/epn/2019203

Showcasing cutting edge physics research to school children and the general public humanizes 'the scientist'. This can help towards removing stereotypes and enabling greater diversity in physics. Here we share some of the lessons learnt and activities conducted at the University of Southampton to achieve this goal.

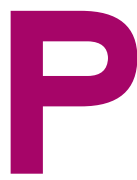

hysics can often be challenging to communicate to a general audience, in part because the concepts can be abstract and far removed from the layperson's everyday experience. The 2018 Nobel Prize in Physics is a prime example of this challenge, as the uses of squeezed light and optical tweezers are not immediately obvious to a non-specialist. Despite this, effective communication of such non-intuitive concepts to the general public is increasingly being identified as a social obligation by research funding bodies [1].
The recent Nobel Prize also highlighted the limited diversity in Physics, as Professor Donna Strickland was the first female Physics laureate for 55 years. Promoting physics to a broad range of people will help ensure that there is a range of talent driving new ideas and innovation $[2,3]$. Outreach and public engagement offer useful routes to communicate with diverse audiences and can begin to change the perceived stereotypes of physicists, making career paths in physics more accessible to all.
$\Delta$ Light Express Total Internal Reflection Demonstration (Credit photograph: Dr Sam Berry) 
Over the last two decades the University of Southampton has operated a dedicated programme of optics and photonics Outreach and Public Engagement (OPE). The programme, run by a team of volunteers and dedicated staff, reaches approximately 5,000 members of the public, pupils and teachers, annually. Stakeholders are both internal to the University and external organisations, all of whom provide leadership, event funding and partnership opportunities. In this article we discuss some of our initiatives, outline lessons we have learned, benefits received and the importance of evaluation.

The development of any new outreach workshop or public engagement event by our programme team starts by us asking one question: "what are we trying to achieve with this event?" Determining where the motivation for the activity lies directs our approach in planning an activity.

The design of an OPE event is dictated by the type of audience. Targeting audiences from under-served groups is important to increase both the number of people in physics and the diversity of backgrounds, but reaching a new audience can often be challenging. Several of our OPE initiatives are designed in partnership with the University of Southampton's Widening Participation team, who work to increase the number of students at university from schools with low levels of progression to higher education. Approaching schools can be often difficult due to the numerous pressures on the time and resources of school staff, but this is often even more pronounced in schools in disadvantaged socioeconomic areas. The Widening Participation team at Southampton conducts multiple engagements with the same audience, which is important in raising the "science capital" of the participants. This concept has been introduced to provide a valuable insight into why and how some people participate and engage with science and why some do not [4]. Familiarising people with science and raising their science capital through multiple OPE engagements has been demonstrated as being the one of most effective ways of making a career in science more accessible [5].

In general we, at Southampton, provide a broad range of activities, including interactive lectures for large audiences, smaller-scale hands-on activities for schools and resources and photonics training events for teachers. Examples of our activities include:

The Light Express Roadshow [6], which is an interactive lecture series on photonics, pictured in Figure 1. Light Express is used primarily as a photonics public engagement activity, allowing demonstrators to explain the basic physics behind their research during the show;

The Lightwave Roadshow [7], a set of hands-on experiments and demonstrations delivered primarily to school classes for outreach purposes;

Photonics Explorer kits [8], an outreach activity with school teachers, providing them with training and resources to teach photonics with a kit for an accompanying optics workshop. Each kit can provide materials to teach 200 students (from primary to college age), pictured in Figure 2; The Photon Shop for the 'Light Up Poole' art festival [9]. This places a team of researchers in an empty shop for three nights, to reach new audiences, through hands-on public engagement activities with photonics, physics and astronomy demonstrations, supported by the Institute of Physics (IOP) [10].

The activities we deliver for outreach events are inherently one-directional and are more akin to an informal teaching session about physics and photonics in general. Ideally, we include demonstrations that are

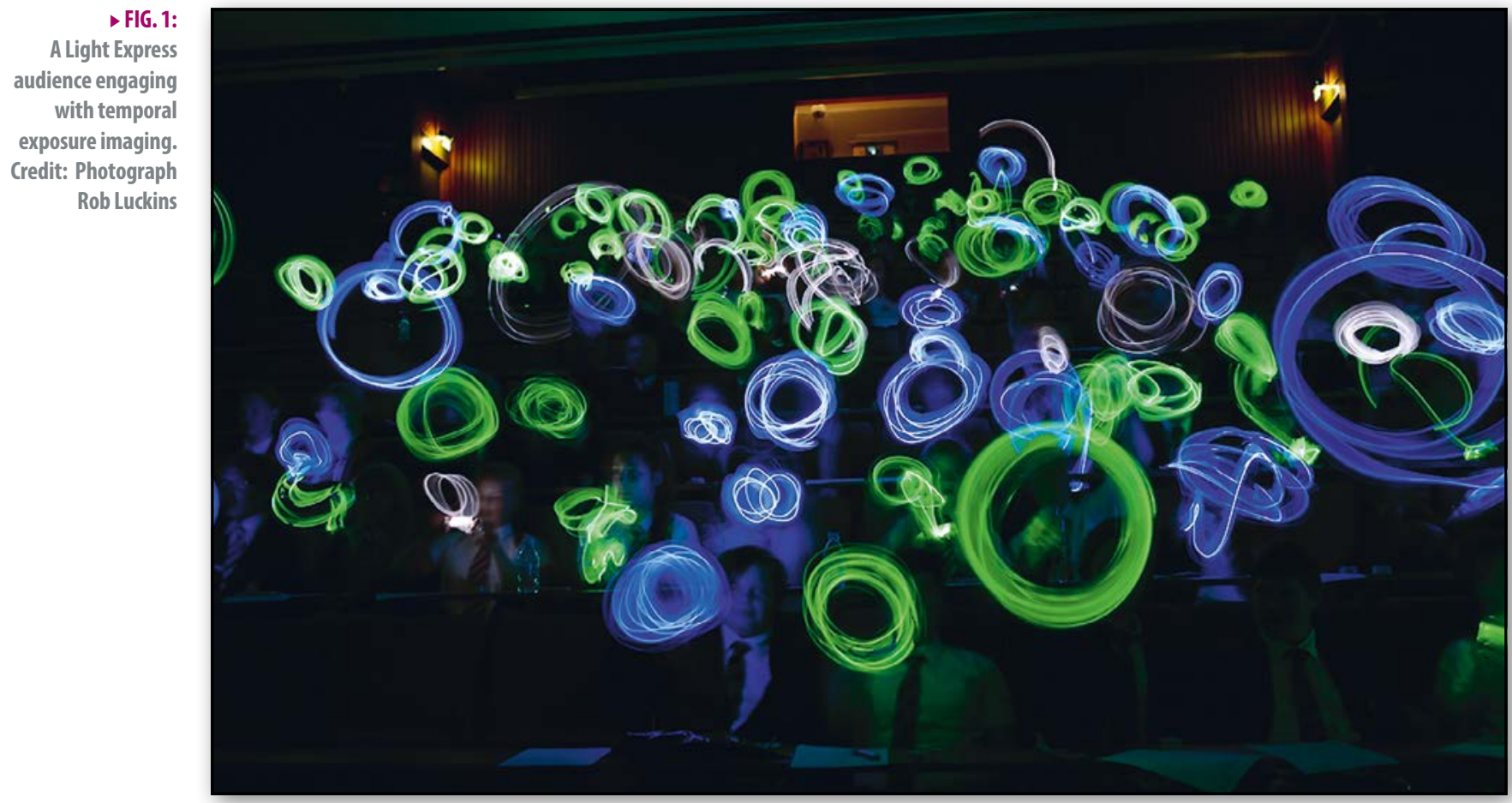


both illustrative and can capture the imagination of the audience; activities that "show" rather than "tell" are better received. For workshops, this does not restrict the activities from being interactive. A good example is the 'Guess the Gas' workshop [11], designed by the Outreach team of the South East Physics Network (SEPnet), which allows students to explore the concepts of spectroscopy by constructing the experimental equipment needed.

Good demonstrations can extend known concepts in a way that they would have not previously have been taught. For example, a common topic for a primary Lightwave activity is for the workshop to begin with a demonstration of a simple reflection from a mirror, perhaps in the context of an Infinity Mirror (shown in Figure 3), then to progress to an explanation of how optical fibres work by many reflections inside a core, and end with a description of how the Internet depends on both reflection and optical fibres. We find that a strong narrative, from observation to theory or from problem to solution, is essential for explaining the relevance and context to audiences. Further examples of inspiring activities can be found from STEM Learning [12] or the Physics Education journal [13].

Public engagement events are approached differently with an objective of having a two-way conversation with an audience. Best practice in Public Engagement would be citizen science projects, such as Aurora Zoo, Southampton's project (currently under review) on the Zooniverse citizen science platform [14]. Zooniverse is a research platform covering many disciplines in which "anyone can contribute to real academic research on their own computer at their own convenience" [15]. Alternatively, Public Engagement activities can provide opportunities for the general public to interact with scientists in an informal environment, such as in our 2018 International Day of Light celebration [11] or during the Pint of Science festival talks which includes discussion about research in pubs and cafes [16].

It is important to note, that not only do the public and schools benefit from OPE. Those delivering activities, whom at Southampton are predominately postgraduate students, benefit greatly from the skills and experience they aquire through the interaction. This experience is invaluable when it comes to job applications and interviews. When interviewed for this article former Lightwave Director Dr Hannah Collins said, "I have often been asked about public engagement at job interviews, and relied on that experience to demonstrate important transferrable skills." These skills can include leadership and team management, event planning, marketing, budgeting and video production, none of which are taught by a typical Physics undergraduate or postgraduate programme.

An obvious benefit to the demonstrator is the development of their communication skills. Every scientist needs to be able to communicate their research effectively. For OPE however, we cannot assume any background

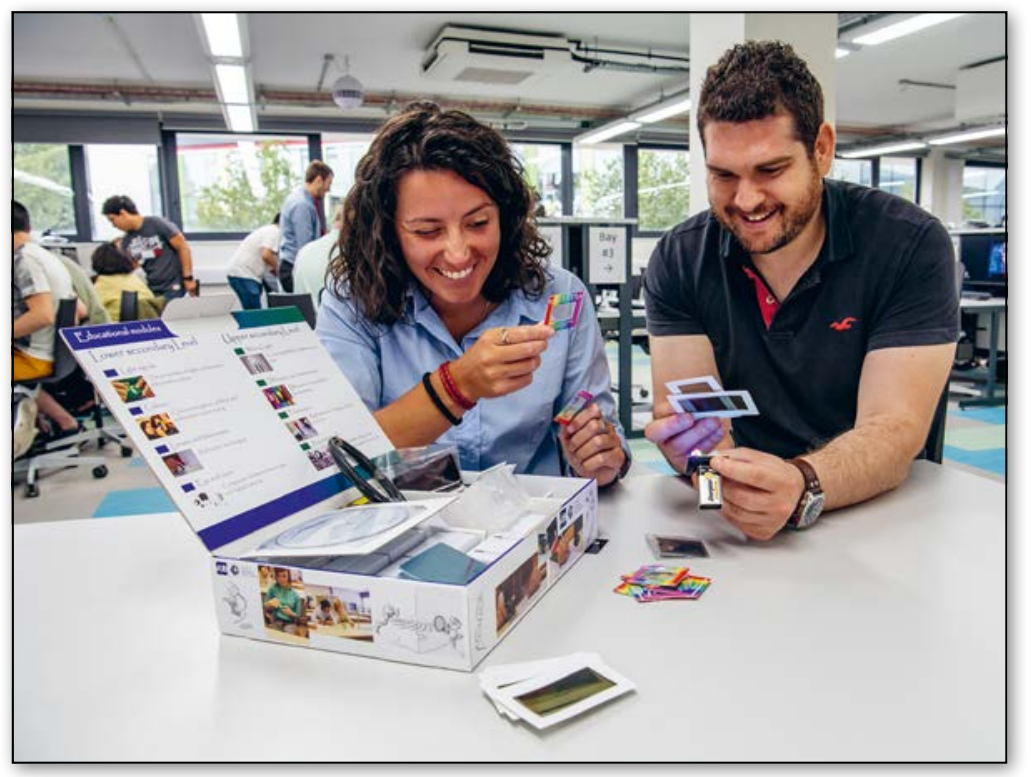

knowledge or even interest on the part of the audience. Demonstrators are challenged to be both succinct and clear in their descriptions of science in ways they would not be through ordinary academic presentations. If researchers leave academia for industry, this particular style of communication is very useful, for example in effectively describing an innovation to a non-technical stakeholder.

Furthermore, student-led OPE activities can provide a valuable opportunity for the students to gain experience in writing grant applications, an essential skill for any career academic, much earlier than they otherwise would in their career path. As part of our activities, students have applied for outreach grants through student chapters of the Optical Society, SPIE and IEEE Photonics Society, as well as through the Institute of Physics and the Royal Academy of Engineering.

The impact of our all our activities is measured in reach (the numbers we have engaged with) and significance for the audience. To determine significance effective evaluation is necessary. Our learning outcomes are based on 'Generic Learning Outcomes' designed for informal education settings [10]. We evaluate to determine participant's enjoyment of activities, whether they have gained any knowledge, and whether we have changed their views or behaviours. It is important to refer back to learning outcomes, to ensure the questions you ask are appropriate in determining whether these outcomes have been fulfilled.

The method of evaluation can vary greatly depending on the type of activity and its delivery. Most evaluation methods consist of completing a form, with perhaps a short knowledge quiz and additional questions on enjoyment and levels of interest in science. The design of the form should be appropriately designed for the audience, for example, to avoid young children becoming bored. Other ideas for evaluation include:

Snowballing: an engaging method to receive feedback. The concept being that instead of long evaluation forms,
\FIG. 2:

Postgraduates demonstrating the Photonics Explorer kit to Teachers, (from EYEst) demonstrating diffraction gratings, Light Emitting Diodes and polarisation principles. Credit: Jon Banfield 
an audience can be asked to write ratings (out of 10) along with the favourite (or least favourite) part of the show onto a piece of paper, which will be screwed up and thrown to the front of the room. This is a suitable method to determine if activities need to be changed to appeal to the age-group.

Social media is also a powerful tool for evaluation for mature audiences; asking an audience to Tweet, can give a clear idea of which parts of an event are capturing the imagination of the public and also provide recognition of the activity for a much wider audience.

Electronic quiz software, such as KAHOOT, enables real time data collection, and makes the evaluation process more time effective.

Running focus groups and interviewing participants can help determine the following; their enjoyment of the activity and whether there has been a change in their knowledge and/or behaviour. Our methods have also included gathering before/after drawings of what a scientist looks like to determine whether scientist stereotypes have been successfully challenged.

There is a strong motivation for university researchers to engage with public audiences, partially promoted through their funding bodies. Such activities have clear benefits for the demonstrators involved, schools and members of the public. Showcasing the cutting edge research to schools and the general public humanizes 'the scientist'. This can help towards perception of science, working towards greater diversity in the range of people entering physics. Through OPE, researchers not only share what they know, but can also learn from the public and bring that learning into research, design and decision-making. It is important moving forward that we not only assess which resources and techniques work well, but to then share these ideas so that the quality and effectiveness of our efforts can be maximised. Help us

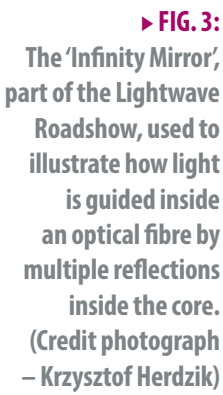

> FIG. 3:

The 'Infinity Mirror', part of the Lightwave Roadshow, used to illustrate how light is guided inside an optical fibre by multiple reflections inside the core. (Credit photograph - Krzysztof Herdzik)

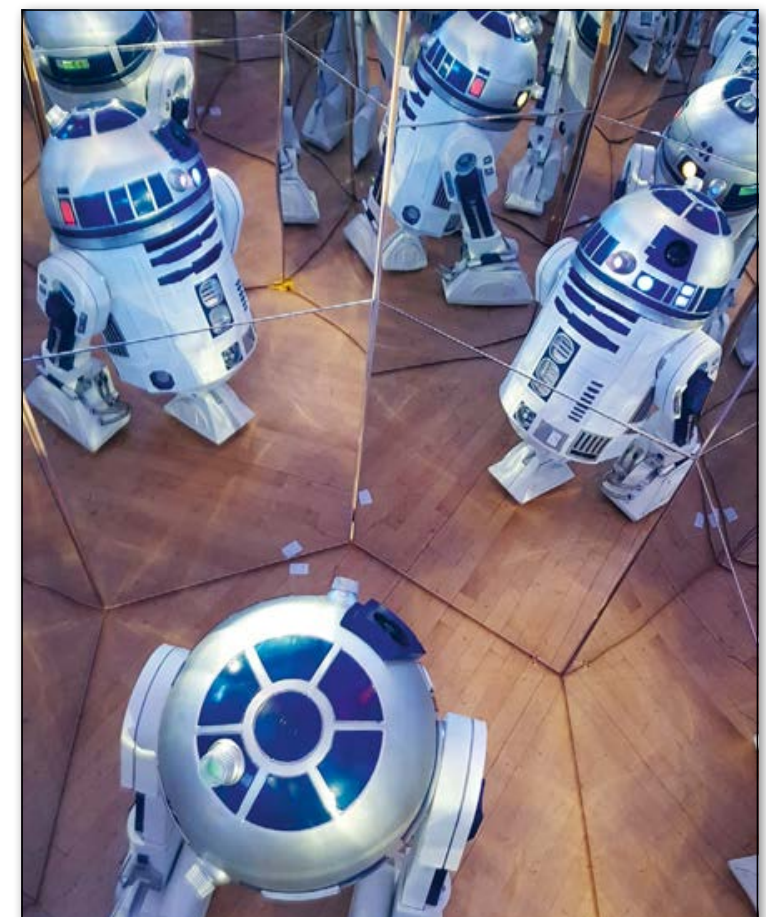

work towards this goal by contributing to the discussion through Twitter: \#ANobelCause.

\section{About the Authors}

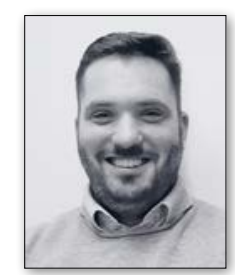

Christopher Holmes is a Senior Enterprise Fellow at the Optoelectronics Research Centre, University of Southampton leading activity in Optoavionics. Holmes is a STEM Ambassador and was the 2005/06 Lightwave Director.

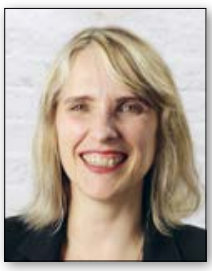

Pearl John is the Public Engagement Leader in Physics and Astronomy at the University of Southampton. John has worked in Photonics education and outreach for over 25 years, specialising in teaching young people holography. She is an internationally acclaimed artist.

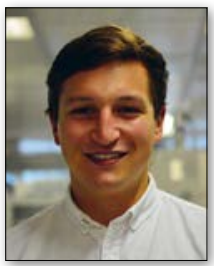

Callum Stirling received his MSci in Physics from the University of Birmingham in 2017, before joining the Optoelectronics Research Centre, University of Southampton. Stirling is a postgraduate researcher specialising in mid-infrared silicon photonics and a Director of the Lightwave Roadshow.

\section{References}

[1] https://www.ukri.org/public-engagement/

[2] M. Nathan and N. Lee. Cultural Diversity, Innovation, and Entrepreneurship: Firm-level Evidence from London, Economic Geography 89(4), 367-394 (2013)

[3] C. Díaz-García, A. González-Moreno and F. Jose Sáez-Martínez. Gender diversity within R\&D teams: Its impact on radicalness of innovation, Innovation 15(2), 149-160 (2013)

[4] https://transformingpractice.sciencemuseum.org.uk/ what-is-science-capital/

[5] www.stem.org.uk

[6] www.lightexpress.soton.ac.uk

[7] N.H.L. Wong, M.T. Posner, P.V. John, 'The Lightwave Programme and Roadshow: An Overview and Update', ETOP Proceedings, paper OUT02, (2015)

[8] www.photonics4all.eu

[9] https://lightuppoole.co.uk/

[10] www.iop.org/about/grants/outreach/page_38843.html

[11] C. J. Stirling et al. Student-led outreach and public engagement activities at the University of Southampton to celebrate the inaugural International Day of Light, Proc. SPIE 10741 (2018).

[12] www.zooniverse.org/projects/dwhiter/aurora-zoo

[13] http://iopscience.iop.org/journal/0031-9120

[14] https://pintofscience.co.uk/

[15] www.zooniverse.org/

[16] www.kcl.ac.uk/sspp/departments/education/research/ ASPIRES/Index.aspx 